\title{
Metabolism of sucrose in a non-fermentative Escherichia coli under oxygen limitation
}

\author{
Karel Olavarria ${ }^{1}$ (D) $\cdot$ Albert Fina $^{1} \cdot$ Mariana I. Velasco ${ }^{1} \cdot$ Mark C. M. van Loosdrecht ${ }^{1} \cdot$ Sebastian Aljoscha Wahl $^{1}$
}

Received: 25 January 2019 / Revised: 10 May 2019 / Accepted: 11 May 2019 / Published online: 31 May 2019

(C) The Author(s) 2019

\begin{abstract}
Biotechnological industry strives to develop anaerobic bioprocesses fueled by abundant and cheap carbon sources, like sucrose. However, oxygen-limiting conditions often lead to by-product formation and reduced ATP yields. While by-product formation is typically decreased by gene deletion, the breakdown of oligosaccharides with inorganic phosphate instead of water could increment the ATP yield. To observe the effect of oxygen limitation during sucrose consumption, a non-fermentative Escherichia coli K-12 strain was transformed with genes enabling sucrose assimilation. It was observed that the combined deletion of the genes $a d h E, a d h P, m h p F$, ldhA, and $p t a$ abolished the anaerobic growth using sucrose. Therefore, the biomassspecific conversion rates were obtained using oxygen-limited continuous cultures. Strains performing the breakdown of the sucrose by hydrolysis (SUC-HYD) or phosphorolysis (SUC-PHOSP) were studied in such conditions. An experimentally validated in silico model, modified to account for plasmid and protein burdens, was employed to calculate carbon and electron consistent conversion rates. In both strains, the biomass yields were lower than expected and, strikingly, SUC-PHOSP showed a yield lower than SUC-HYD. Flux balance analyses indicated a significant increase in the non-growth-associated ATP expenses by comparison with the growth on glucose. The observed fructose-1,6-biphosphatase and phosphoglucomutase activities, as well as the concentrations of glycogen, suggest the operation of ATP futile cycles triggered by a combination of the oxygen limitation and the metabolites released during the sucrose breakdown.
\end{abstract}

Keywords Sucrose phosphorolysis $\cdot$ Flux balance analysis $\cdot$ Plasmid burden $\cdot$ Data reconciliation $\cdot$ Carbon storage regulator A

\section{Introduction}

The design of efficient and sustainable biotechnological processes demands the maximization of product titer, production rate, and yield, and the reduction of fixed and variable costs (Porro et al. 2014). All these demands call for the development of anaerobic processes as opposed to aerobic cultivations

Karel Olavarria and Albert Fina contribute equally to this work.

Electronic supplementary material The online version of this article (https://doi.org/10.1007/s00253-019-09909-6) contains supplementary material, which is available to authorized users.

Karel Olavarria

K.OlavarriaGamez@tudelft.nl

Sebastian Aljoscha Wahl

S.A.Wahl@tudelft.nl

1 Department of Biotechnology, Applied Sciences Faculty, Delft University of Technology, van der Maasweg 9, 2629, HZ Delft, The Netherlands
(Cueto-Rojas et al. 2015). Further reduction of variable costs is obtained when using abundant, cheap feedstocks like sucrose (Marques et al. 2016).

Although E. coli K-12-derived strains can anaerobically grow using sucrose as the sole carbon source (Hoefel et al. 2012), the bio-energetic impact of the oxygen limitation while using this carbon source has not been studied in detail. This kind of analysis is required to improve the efficiency of the bioprocesses fueled by sucrose.

On the other hand, despite the advantages of the anaerobic conversions, the generation of by-products and the decrease in the ATP yield under such conditions often represent important obstacles. Some of the by-products generated by E. coli are weak acids. As well as diverting carbon otherwise available for the generation of the target product(s), weak acids could be transported cyclically across the membrane, leading to ATP losses (Axe and Bailey 1995). Moreover, the presence of such by-products could trigger physiological responses whose effects might be difficult to anticipate and quantify (Roe et al. 2002). Therefore, the suppression of these by-products is 
usually beneficial for the bioprocesses, and gene deletion is the tool typically used to achieve this goal (Jantama et al. 2008; Jian et al. 2010; Wang et al. 2012). However, the consequences of such deletions on the redox-balancing mechanisms need to be considered.

Regarding the decrease in ATP yield, it is important to highlight that this could make the generation of some biotechnologically relevant products unfeasible. One of the strategies employed to increase the ATP yield is the use of phosphorylases (also known as phosphorolases) to catalyze the breakdown of oligosaccharides (de Kok et al. 2011; Marques et al. 2018). The incorporation of inorganic phosphate during the breakdown of the glycosidic bonds means a saving in the ATP consumed to phosphorylate the monosaccharides fueling the glycolysis (Zhang and Lynd 2005). Therefore, in addition to its abundance and low price, using sucrose as substrate has the potential to enhance the ATP yield during (anaerobic) fermentations in E. coli.

Different oligosaccharide phosphorylases have been found (Mukherjee et al. 2018) or have been heterologously expressed in E. coli, including the sucrose phosphorylase from Bifidobacterium adolescentis (De Bruyn et al. 2015). Nevertheless, to the best of our knowledge, there are no published studies about the impact on the biomass yield of implementing oligosaccharide phosphorolysis in E. coli.

The aim of this study was the quantitative analysis of the cellular energetics, especially biomass yield, of $E$. coli $\mathrm{K}-12$ under conditions relevant for the biotechnological industry: using a cheap and abundant carbon source (sucrose), with a limited oxygen supply, and using genetically modified cells to diminish by-product generation. Having this objective, we expressed genes enabling the sucrose uptake and hydrolysis/phosphorolysis (Fig. 1) in a mutant E. coli $\mathrm{K}-12$ with deletions reducing its capacity to generate by-products such as ethanol, acetate, lactate, and formate. The biomass-specific conversion rates of mutant strains breaking down the sucrose using water or inorganic phosphate were compared.

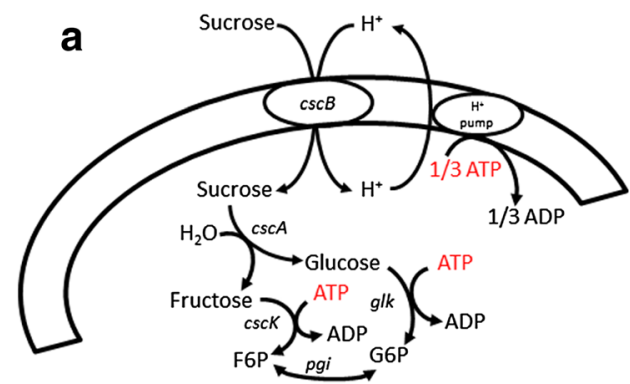

Fig. 1 Functions of the proteins encoded by the genes $\csc A, \csc B, \csc K$, and $s u c P$. The differences in the spent ATP to obtain fructose-6-phosphate depending on the mechanism to break down the sucrose (water in a, phosphate in b) are also highlighted. Some enzymes and the sucrose $/ \mathrm{H}^{+}$

\section{Materials and methods}

\section{Construction of the strains}

The characteristics of the hosting strains are summarized in the Table 1 . The hosting strain TUD58 has a DE3 insertion on the lac $Z$ locus (Tseng et al. 2009) and deletions of the genes $a d h E$ (encoding for an alcohol dehydrogenase), $a d h P$ (encoding for another alcohol dehydrogenase), ldhA (encoding for the lactate dehydrogenase), pta (encoding for the phosphotransacetylase), and $m h p F$ (encoding for an acetaldehyde dehydrogenase). These deletions had already been performed, using the method described by Wanner and Datsenko (Datsenko and Wanner 2000). The resulting strain after these deletions was kindly donated by Ana Sofia Araújo Ferreira and Isabel Rocha from University of Minho, Portugal. Details of the construction of the plasmids pUC19$\csc K B$, pUC19-csc $A K B$, and pUC19-cscKB-sucP are described in Supplementary Material 1. The sequences of the employed primers are listed in Table S1. The supplementary files $p U C 19-c s c K B . g b, p U C 19-c s c A K B . g b$, and $p U C 19$ $\csc K B$-sucP.g $b$ contain the DNA sequences of these plasmids. These plasmids were introduced, by electroporation, into cells of the strains E. coli K-12 MG1655 and TUD58.

\section{Cultures in batch}

Mineral medium supplemented with $15 \mathrm{~g} / \mathrm{L}$ of sucrose or $15 \mathrm{~g} /$ $\mathrm{L}$ of glucose was used for the cultures in batch. The content of one liter of mineral medium (excluding the carbon source) was $5 \mathrm{~g}$ of $\left(\mathrm{NH}_{4}\right) \mathrm{SO}_{4}, 2 \mathrm{~g} \mathrm{KH} \mathrm{PO}_{4}, 0.5 \mathrm{~g} \mathrm{MgSO} \cdot 7 \mathrm{H}_{2} \mathrm{O}$, $0.5 \mathrm{~g} \mathrm{NaCl}, 2 \mathrm{~g} \mathrm{NH}_{4} \mathrm{Cl}, 0.001 \mathrm{~g}$ of thiamine, and $2 \mathrm{~mL}$ of a trace-element solution prepared as previously described (Verduyn et al. 1992). All the components of the medium, excluding the carbon source, the thiamine, and the trace elements, were mixed and the $\mathrm{pH}$ of the medium was adjusted to 7.0 using a solution of $2 \mathrm{M} \mathrm{KOH}$ before sterilization in autoclave. After autoclaving, the thiamine, the trace elements, and the carbon source were added to the sterile media using a

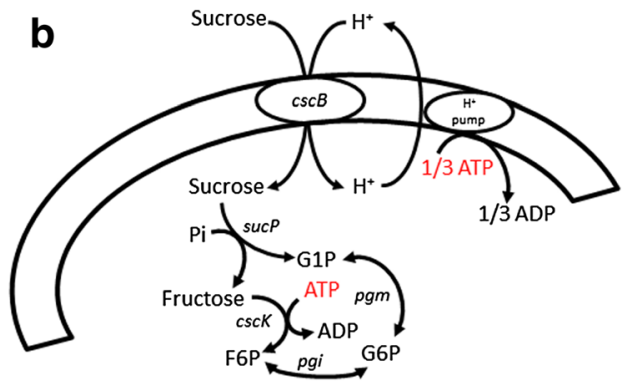

symporter are represented with the names of the corresponding encoding genes. F6P, fructose-6-phosphate; G6P, glucose-6-phosphate; G1P, glucose-1-phosphate; $\mathrm{Pi}$, inorganic phosphate 
Table 1 E. coli $\mathrm{K}-12$ strains employed in this study

\begin{tabular}{|c|c|c|}
\hline Strain & Background & Plasmid \\
\hline MG1655 & $\begin{array}{c}(\mathrm{F}-\lambda-i l v G-r f b-50 r p h-1) \mathrm{DSM} 498, \text { ATCC } 23716, \mathrm{CECT} 7619, \mathrm{CIP} \\
110067\end{array}$ & - \\
\hline TUD58 & $(\mathrm{F}-\lambda-i l v G-r f b-50 r p h-1$ (DE3)) $\Delta a d h E \Delta a d h P \Delta l d h A \Delta p t a \Delta m h p F$ & - \\
\hline TUD37 & $(\mathrm{F}-\lambda-i l v G-r f b-50 r p h-1)$ & pUC19-cscBK \\
\hline TUD66 & $(\mathrm{F}-\lambda-i l v G-r f b-50 r p h-1)$ & pUC19-cscAKB \\
\hline TUD69 & $(\mathrm{F}-\lambda-i l v G-r f b-50 r p h-1)$ & pUC19-cscKB-sucP \\
\hline TUD38 & $(\mathrm{F}-\lambda-i l v G-r f b-50 r p h-1(\mathrm{DE} 3)) \Delta a d h E \Delta a d h P \Delta l d h A \Delta p t a \Delta m h p F$ & pUC19-cscBK \\
\hline SUC-HYD & $(\mathrm{F}-\lambda-i l v G-r f b-50 r p h-1$ (DE3)) $\Delta a d h E \Delta a d h P \Delta l d h A \Delta p t a \Delta m h p F$ & pUC19-cscAKB \\
\hline SUC-PHOSP & $(\mathrm{F}-\lambda-i l v G-r f b-50 r p h-1(\mathrm{DE} 3)) \Delta a d h E \Delta a d h P \Delta l d h A \Delta p t a \Delta m h p F$ & pUC19-cscKB-sucP \\
\hline
\end{tabular}

syringe coupled to a $0.22-\mu \mathrm{m}$ polyvinyl difluoride (PVDF) filter (Millipore, Germany). All the experiments in batch were performed in triplicate.

For the growth experiments under anaerobic conditions, the medium was supplemented with $0.24 \mathrm{mg} / \mathrm{L}$ of $\mathrm{Na}_{2} \mathrm{SeO}_{3}$ $\times 5 \mathrm{H}_{2} \mathrm{O}, 0.28 \mathrm{mg} / \mathrm{L} \mathrm{Na}_{2} \mathrm{MoO}_{4}$, and $2.3 \mathrm{mg} / \mathrm{L} \mathrm{Ni}\left(\mathrm{NO}_{3}\right)_{2} \times 6$ $\mathrm{H}_{2} \mathrm{O}$ to provide the metals required for the activity of the formate hydrogen lyase (Soini et al. 2008), and it was also supplemented with $50 \mathrm{mM}$ of 3-(N-morpholino)propanesulfonic acid (MOPS) to increase the buffering capacity of the medium. For these experiments, sealed bottles were used. Once the medium was inside the sealed bottles, a continuous stream of sterile compressed nitrogen was sparged into the bottles to remove the oxygen from both the liquid and the headspace. The sealed bottles were inoculated with cells taken from an aerobic preinoculum culture grown on the same medium. The initial optical density of the cultures at $600 \mathrm{~nm}$ was 0.1 .

\section{Continuous cultures}

The growth medium employed for the continuous cultures was the same as that employed for the cultures in batch. The continuous cultures were performed at dilution rates of 0.05 $\mathrm{h}^{-1}$ and the aeration rate was kept constant at 0.55 v.v.m. The stirring speed was initially set to 500 r.p.m. to achieve a steady state in fully aerobic conditions (dissolved oxygen tension above 50\%) (Supplementary Material 2, Table S2, Fig. S2 and supplementary file calculating required stirring.xls).

The stirring rate was gradually decreased to achieve the oxygen limitation. The signals of dissolved oxygen tension and $\mathrm{CO}_{2}$ in the outgas were followed until non-stable fluctuations in $\mathrm{CO}_{2}$ in the outgas were observed. Once the oxygenlimited steady states were approached, samples of broth, biomass, and cell-free extracellular media (filtrates) were obtained at two different times during the same steady state. Continuous cultures were assumed to be in steady state when the signal corresponding to the $\mathrm{CO}_{2}$ in the outgas remained stable for at least two culture volume changes. Other details of the setup, operation, sampling, and sample analyses of the continuous cultures are explained in Supplementary Materials 3 and 4.

\section{Enzymatic assays}

Cells harvested from batch cultures during the exponential growth phase or from the continuous cultures were used for determination of the specific activities. Three different assays were performed: sucrose phosphorylase (SucP), phosphoglucomutase, and fructose-1,6-bisphosphatase. These three experiments were coupled assays leading to the production of NADPH from NADP ${ }^{+}$. Thus, the production of NADPH, which was followed spectrophotometrically at $340 \mathrm{~nm}$, was used to calculate the reaction rate. All the enzymatic assays were performed at $37^{\circ} \mathrm{C}$. The choice of the buffer used in each case was related to the stability of the substrates and the products, while the concentration of the substrates and the auxiliary enzymes was always high enough to ensure maximum activity of the studied enzymes under the conditions of the assays. In each case, the background activity observed when no substrate is added to the reaction mixture was subtracted from the total activity. Moreover, we checked that no changes in the signal were observed before addition of the cell-free extracts. The details of each enzymatic assay are described in Supplementary Material 5.

Data availability

All the relevant data and scripts are provided as supplementary material.

\section{Results}

\section{Expression of sucP enabled growth on sucrose, but deletions of $a d h E, a d h P, I d h A, p t a$, and $m h p F$ suppressed anaerobic growth}

For enabling the sucrose uptake and catabolism in E. coli $\mathrm{K}-12$, we chose the episomal expression of the foreign genes $\csc B$ and $\csc K$ from $E$. coli W combined with the 
cscA gene from $E$. coli $\mathrm{W}$ or the sucP gene from $B$. adolescentis. The processes enabled by the proteins encoded by these genes are presented in Fig. 1. Although growth on sucrose had been reported after expressing only the $\csc$ A gene (Sahin-Toth et al. 1999; Tsunekawa et al. 1992), the observed growth rates were much lower than the obtained expressing a sucrose transporter (Bruschi et al. 2012). Therefore, our rationale to include the expression of the $\operatorname{cscBK}$ genes next to the expression of $\csc A$ or $s u c P$ was to increase the active uptake and quick funneling of the sucrose into fructose-6-phosphate.

The hosting cells were from an E. coli K-12 mutant strain with multiple gene deletions ( $\triangle a d h E \Delta a d h P$ $\triangle l d h A \Delta p t a \Delta m h p F)$. These deletions were previously performed with the aim of minimizing the formation of by-products (acetate, lactate, and ethanol) under oxygenlimited conditions.

Batch cultures at $37^{\circ} \mathrm{C}$, in aerobic or anaerobic conditions, were monitored for up to seven days. The different outcomes are shown in Table 2. In the strains carrying the plasmid pUC19-cscKB, no growth on sucrose was observed. This result confirms that, without the introduction of some gene encoding for an enzyme catalyzing the breakdown of the sucrose-either a hydrolase or a phosphorylase-growth of E. coli $\mathrm{K}-12$ on sucrose is not possible.

Regarding the growth/no-growth pattern observed under anaerobiosis, it is possible to conclude that the deletions of the genes $a d h E$, adhP, ldhA, $p t a$, and $m h p F$ were sufficient to abolish growth under the tested conditions. Because we were interested in the study of the sucrose metabolism under oxygen limitation while maintaining low (or zero) by-product formation rates, our chosen approach was the study of the biomass-specific consumption and production rates in strains with the $\triangle a d h E \Delta a d h P \Delta l d h A \Delta p t a \Delta m h p F$ deletions using oxygen-limited continuous cultures.

\begin{tabular}{llll}
\cline { 2 - 3 } $\begin{array}{l}\text { Table } 2 \text { Growth/no } \\
\text { growth using sucrose as } \\
\text { the sole carbon source, in } \\
\text { aerobiosis and }\end{array}$ & Strain & Condition & Outcome \\
\cline { 2 - 4 } $\begin{array}{l}\text { anaerobiosis, for the } \\
\text { different recombinant }\end{array}$ & TUD37 & Aerobic & No growth \\
strains obtained & TUD66 & Aerobic & Growth \\
combining two different & TUD37 & Aerobic & Growth \\
genetic backgrounds & TUD66 & Anaerobic & No growth \\
with three different & TUD69 & Anaerobic & Growth \\
plasmids & TUD38 & Aerobic & No growth \\
& SUC-HYD & Aerobic & Growth \\
& SUC-PHOSP & Aerobic & Growth \\
& TUD38 & Anaerobic & No growth \\
& SUC-HYD & Anaerobic & No growth \\
& SUC-PHOSP & Anaerobic & No growth \\
\cline { 2 - 4 } & & &
\end{tabular}

\section{Construction of an ad hoc in silico metabolic model}

For calculation of the biomass-specific conversion rates and possible flux distributions, the biomass composition and the network stoichiometry are very relevant (Dikicioglu et al. 2015). For this reason, the metabolic model constructed by Taymaz-Nikerel and co-workers (Taymaz-Nikerel et al. 2010) was adapted for the design and analysis of the continuous cultures on sucrose (Supplementary Material 2 and the supplementary files iKOGmaker.m, iKOG.mat, and Ecolicore.mat). This model was chosen because (i) it contains experimentally validated estimates for the energetic parameters $\mathrm{P} / \mathrm{O}$ ratio $(\delta$, in mol of ATP/mol of $\mathrm{O})$, growth-dependent ATP expense ( $K x$, in mol of ATP/C-mol biomass $)$, and ATP expense for growth-independent maintenance $\left(m_{\text {ATP }}\right.$ in mol of ATP/C-mol ${ }_{\text {biomass }} / \mathrm{h}$ ); and (ii) it contains experimentally determined biomass composition data for the employed dilution rate. However, some modifications were implemented in the original model to represent the genetic modifications present in the constructed strains. For example, the fluxes through the reactions catalyzed by the ethanol dehydrogenase, acetaldehyde dehydrogenase, lactate dehydrogenase, and phosphotransacetylase were set to zero to represent the effect of the gene deletions $\triangle a d h E, \triangle a d h P, \Delta m h p F, \Delta l d h A$, and $\triangle p t a$. Moreover, the in silico model was further adapted to account for the plasmidial DNA and plasmid-encoded protein burdens in the mutant strains. For these corrections, the DNA and protein sequences, the contribution to the cellular weight of the plasmid-encoded beta-lactamase, and the observed specific sucP activities $\left(12.41 \pm 0.45 \mu \mathrm{mol} / \mathrm{min} / \mathrm{mg}_{\text {soluble protein }}\right.$ in SUC-PHOSP; $0.05 \pm 0.04 \mu \mathrm{mol} / \mathrm{min} / \mathrm{mg}_{\text {soluble protein }}$ in SUCHYD) were considered.

Flux balance analysis (FBA) using the software Constraints Based Reconstruction and Analysis (COBRA, version 2.0.6) (Schellenberger et al. 2011) for MATLAB (MathWorks, USA) was employed for both the experimental design and the estimation of the metabolic fluxes in the cells grown in the continuous cultures. To predict the metabolic fluxes in the cells growing in the continuous cultures, minimization of the sucrose uptake rate was used as the objective function (see supplementary file predictionsHYDvSSUCP.m). To estimate the actual metabolic fluxes, reconciled specific consumption and production rates were employed as constraints, and maximization of the ATP production was used as the objective function. Maximization of ATP production has been reported as a reliable objective function when cells are growing in continuous cultures (Schuetz et al. 2007; van Gulik and Heijnen 1995).

\section{Sucrose utilization under oxygen-limiting conditions}

The experimentally determined specific consumption/ production rates with their associated errors (Supplementary 
Material 6 and supplementary files qratesHydrolase.m, qratesPhosphorylase.m, SucHYD.xls, and SUCP.xls) formed the input used to estimate reconciled rates (Table 3; and supplementary files data_reconciliation_Hydrolase.m, data_reconciliation_SucP.m, and reconciled rates.xls), consistent with the carbon and electron conservation principle. Results obtained by other groups while studying E. coli MG1655 and E. coli mutants with gene deletions similar to the employed in this study, using continuous cultures, are included in Table 3 for comparison.

The carbon and electron recoveries were above $94 \%$ (Supplementary Material 7, Supplementary Material 9, Table S3, Table S6, Table S7, Table S8, and Table S9). Considering the extracellular concentrations measured for sucrose, formate, acetate, lactate, pyruvate, and succinate, it was possible to account for $90-95 \%$ of the total organic carbon (TOC) present in the filtrate (cell-free) samples obtained from the bioreactors (Supplementary Material 7, Table S4). For this reason, it was assumed that no other metabolites, beyond the measured ones, were produced in meaningful amounts.

Sabri and co-workers observed secretion of fructose into the medium in an E. coli W mutant strain where the $\csc K$ gene was knocked out (Sabri et al. 2013). However, we did not detect glucose or fructose in the samples from extracellular medium (data not shown). The heterologous expression of $\csc K$ plus the native hexokinase activity probably guaranteed a quick phosphorylation of the fructose resulting from the sucrose hydrolysis/phosphorolysis, preventing its accumulation and leakage to the extracellular medium.

The by-product generation rates represented similar percentages of the consumed carbon (10\% and 12\%) and electrons (9\% and $11 \%$ ) in SUC-HYD and SUC-PHOSP, respectively. However, some small differences in the by-product profiles were observed between the strains (Table 3 ).

To assess the potential impact of phosphate on the observed phenotypes, the extracellular concentrations of phosphate were measured. Results indicated similar concentrations in the cultures of SUC-PHOSP $(2.6 \mathrm{mM})$ and SUC-HYD $(2.2$ $\mathrm{mM})$. These values are far above the critical concentration triggering the pho regulon ( $4 \mu \mathrm{M}$, (Lamarche et al. 2008)) and should be saturating for all the known phosphate transporters (Harris et al. 2001; Willsky and Malamy 1980). Therefore, extracellular phosphate concentrations should not have affected the phosphate uptake.

Strikingly, the biomass yield observed for SUC-PHOSP was lower than the observed for SUC-HYD (Table 3 vs Fig.

Table 3 Reconciled specific rates during the growth in oxygen-limiting continuous cultures. For comparison, previously published data of different E. coli, growing on continuous cultures, are included

\begin{tabular}{|c|c|c|c|c|c|c|}
\hline & SUC-HYD & SUC-PHOSP & $\begin{array}{l}\text { K-12 } \\
\text { MG16551 }\end{array}$ & $\begin{array}{l}\text { GJT001 } \\
\text { (wild type) }^{2}\end{array}$ & $\begin{array}{l}\text { YBS125 } \\
(\Delta a c k A \Delta p t\end{array}$ & $\begin{array}{l}\text { YBS132 }(\Delta a c k A \\
\Delta p t a \Delta l d h A)^{2}\end{array}$ \\
\hline $\begin{array}{c}\text { Oxygen availability/carbon } \\
\text { source(s) }\end{array}$ & $\begin{array}{l}\mathrm{O}_{2} \\
\underset{\text { limiting/Suc- }}{\text { rose }}\end{array}$ & $\begin{array}{l}\mathrm{O}_{2} \\
\underset{\text { limiting/Suc- }}{\text { rose }}\end{array}$ & $\begin{array}{l}\text { Fully } \\
\text { aerobic/Gluc- } \\
\text { ose }\end{array}$ & $\begin{array}{c}\text { Anaerobic/LB plus } \\
\text { glucose }\end{array}$ & $\begin{array}{c}\text { Anaerobic/LB plus } \\
\text { glucose }\end{array}$ & $\begin{array}{c}\text { Anaerobic/LB plus } \\
\text { glucose }\end{array}$ \\
\hline \multicolumn{7}{|l|}{ Specific rates $\left(\mathrm{mmol} / \mathrm{g}_{\mathrm{CDW}} / \mathrm{h}\right)$} \\
\hline Glucose & - & - & -0.77 & 3.55 & 5.39 & 7.24 \\
\hline Sucrose & $\begin{array}{c}-0.4157 \pm \\
0.0054\end{array}$ & $\begin{array}{r}-0.505 \pm \\
0.0117\end{array}$ & - & - & - & - \\
\hline $\mathrm{O}_{2}$ & $\begin{array}{c}-2.3177 \pm \\
0.0298\end{array}$ & $\begin{array}{c}-3.3428 \pm \\
0.1259\end{array}$ & $-1.97 *$ & - & - & - \\
\hline $\mathrm{CO}_{2}$ & $2.4354 \pm 0.0295$ & $3.3907 \pm 0.1268$ & $2.19 *$ & n.i. & n.i. & n.i. \\
\hline Formate & 0 & $0.0348 \pm 0.0072$ & n.d. & 5.89 & 0.56 & 12.1 \\
\hline Acetate & $0.0036 \pm 0.0002$ & $0.0066 \pm 0.0004$ & n.d. & 3.03 & 0.15 & 0.23 \\
\hline Lactate & $0.0225 \pm 0.0007$ & $0.0129 \pm 0.0008$ & n.d. & 0.18 & 8.29 & 0.05 \\
\hline Pyruvate & $0.0372 \pm 0.0076$ & $0.1119 \pm 0.0185$ & n.d. & 0 & 0.25 & 0.89 \\
\hline Succinate & $0.0773 \pm 0.0127$ & $0.0831 \pm 0.0132$ & n.d. & 0.85 & 1.16 & 0.43 \\
\hline Ethanol & n.d. & n.d. & n.d. & 2.86 & 0.41 & 11.9 \\
\hline \multicolumn{7}{|l|}{ Other parameters } \\
\hline Biomass $\left(\mathrm{h}^{-1}\right)$ & $0.0503 \pm 0.0005$ & $0.0468 \pm 0.0005$ & $0.049 *$ & 0.1 & 0.1 & 0.1 \\
\hline Yield $\left(\mathrm{g}_{\mathrm{CDW}} / \mathrm{g}_{\text {substrate }}\right)$ & $0.3535 \pm 0.0057$ & $0.2708 \pm 0.0069$ & $0.426^{*}$ & 0.156 & 0.103 & 0.077 \\
\hline $\begin{array}{l}\text { Yield } \\
\left(\mathrm{C}-\mathrm{mol}_{\text {biomass }} / \mathrm{C}-\mathrm{mol}_{\text {substr- }}\right. \\
\text { ate })\end{array}$ & $0.4125 \pm 0.0067$ & $0.3188 \pm 0.0081$ & $0.522^{*}$ & 0.191 & 0.13 & 0.094 \\
\hline
\end{tabular}

n.d. not detected, n.i. not informed

${ }^{1}$ Data from Taymaz-Nikerel and co-workers (Taymaz-Nikerel et al. 2010). It was considered $\mathrm{M}_{\mathrm{w}}{ }^{\mathrm{CmolX}}=24.56 \mathrm{~g}_{\mathrm{CDW}} / \mathrm{CmolX}$

${ }^{2}$ Data from Yang and co-workers (Yang et al. 1999). It was considered $\mathrm{M}_{\mathrm{w}}{ }^{\mathrm{CmolX}}=24.56 \mathrm{~g}_{\mathrm{CDW}} / \mathrm{CmolX}$ 
S1). A deeper analysis, considering the effects of the genetic differences between these strains and the different by-product profiles observed during the growth under oxygen limitations, was performed.

With the in silico model corrected to account for the plasmid and recombinant protein burdens (Supplementary Material 10, supplementary file Biomass composition.xls), using the reconciled specific consumption/production rates as constraints, and setting the production of ATP as the objective to be maximized, the distributions of the metabolic fluxes were calculated using FBA (see supplementary files SucHYD_with_burden.m and SucPHOSP_with_burden.m). The upper and lower boundaries for the experimentally determined fluxes were constrained considering the reconciled errors. The calculated metabolic flux distributions are reported in the supplementary file metabolic fluxes distributions.xls.

Given the estimated biomass-specific conversion rates, the maximum growth-independent ATP consumption (maintenance) in SUC-HYD was $0.117 \mathrm{~mol}$ of ATP/C-molbiomass $/ \mathrm{h}$, while in SUC-PHOSP it was $0.205 \mathrm{~mol}$ of ATP/ $\mathrm{C}-\mathrm{mol}_{\text {biomass }} / \mathrm{h}$. These values are, respectively, $30 \%$ and $130 \%$ higher than the upper limit of the growthindependent maintenance ATP expense of $0.075 \pm$ $0.015 \mathrm{~mol}$ of ATP/C-mol biomass $/ \mathrm{h}$ estimated by TaymazNikerel and co-workers for the E. coli K-12 MG1655 wild type growing on glucose under fully aerobic conditions in a similar growth medium (Taymaz-Nikerel et al. 2010). On the other hand, Gonzalez and co-workers estimated a value of $0.054 \mathrm{~mol}$ of ATP/C-mol $\mathrm{bimass}_{\text {biom }} \mathrm{h}$ for E. coli BW25113 (apparently wrongly typed as BW21135) anaerobically growing on glucose (Gonzalez et al. 2017).

Two important conclusions can be obtained from these calculations: (i) the growth of E. coli K-12-derived strains on sucrose under oxygen-limiting conditions, despite the kind of enzymatic mechanism for the breakdown of the disaccharide, implied a large increase in the ATP expenses generically named "maintenance"; and (ii) because biomass compositions, plasmid and protein burdens, $\mathrm{P} / \mathrm{O}$ ratios, and growth-dependent ATP expenses in SUC-HYD and SUC-PHOSP are similar (Supplementary Material 8, Fig. S3, Fig. S4, Table S5), with the available data it is not possible to unmistakably identify the biological process(es) responsible for the difference between the biomass yields observed in such strains. However, further analyses pointed to an increase in ATP futile cycles triggered by the use of sucrose under oxygen-limiting conditions, being these futile cycles higher in SUC-PHOSP.

\section{Evidence pointing to ATP futile cycles of different extensions in SUC-HYD and SUC-PHOSP}

One of the best-known futile ATP cycles is caused by the simultaneous operation of the reactions catalyzed by the fructose-1,6-bisphosphatase (FBPase) and the phosphofructokinase (PFK). The specific FBPase activities in samples of SUC-HYD and SUC-PHOSP, obtained during the continuous cultures, were measured. The activities from aerobic batch cultures of $E$. coli $\mathrm{K}-12 \mathrm{MG} 1655$ grown on $5 \mathrm{~g} / \mathrm{L}$ of acetate and $5 \mathrm{~g} / \mathrm{L}$ of glucose were included for comparison. The results showed that the activity of FBPase was approximately eight times higher in SUC-PHOSP than in SUC-HYD (Table 4). Remarkably, the specific FBPase activities in SUC-HYD and SUC-PHOSP were 10-70 times higher than those observed in E. coli $\mathrm{K}-12$ MG1655 grown in gluconeogenic conditions, i.e., a situation in which FBPase is required to survive.

In any case, the differences in FBPase activities between SUC-HYD and SUC-PHOSP detected in the enzymatic assay reflect differences in the transcription and/or the post-translational level rather than differences caused by allosteric effects. Therefore, we should expect some difference in the activities of the proteins regulating the sugar metabolism. Following this clue, it has been shown that deletion of the csrA gene (encoding for the carbon storage regulator A, CsrA) leads to overexpression of phosphoglucomutase (PGM) (Sabnis et al. 1995). Because PGM is required in SUC-PHOSP for the conversion of glucose 1phosphate into glucose 6-phosphate (G6P), if CsrA activity in SUC-PHOSP were lower, the consequent increment in PGM activity could be beneficial for the rate of sucrose assimilation in SUC-PHOSP. However, the decrease in CsrA activity also led to an increase in the expression of FBPase and PEP synthase (Sabnis et al. 1995), potentially leading to ATP futile cycles (Fig. 2).

To verify this prediction, the PGM activity was measured in samples of SUC-HYD and SUC-PHOSP taken
Table 4 Specific fructose-1,6biphosphatase activity

\begin{tabular}{llll}
\hline Strain & Growth conditions & Carbon source & $\begin{array}{l}\text { Specific activity } \\
\left(\mathrm{U} / \mathrm{mg}_{\text {soluble protein }}\right)\end{array}$ \\
\hline E. coli $\mathrm{K}-12$ MG1655 & Aerobic shake flask & Glucose & $0.44 \pm 0.08$ \\
E. coli K-12 MG1655 & Aerobic shake flask & Acetate & $0.98 \pm 0.05$ \\
SUC-HYD & chemostat & Sucrose & $9.41 \pm 1.47$ \\
SUC-PHOSP & chemostat & Sucrose & $72.32 \pm 4.27$ \\
\hline
\end{tabular}

$1 \mathrm{U}=\mu \mathrm{mol}_{\mathrm{NADPH}} / \mathrm{min}$ 

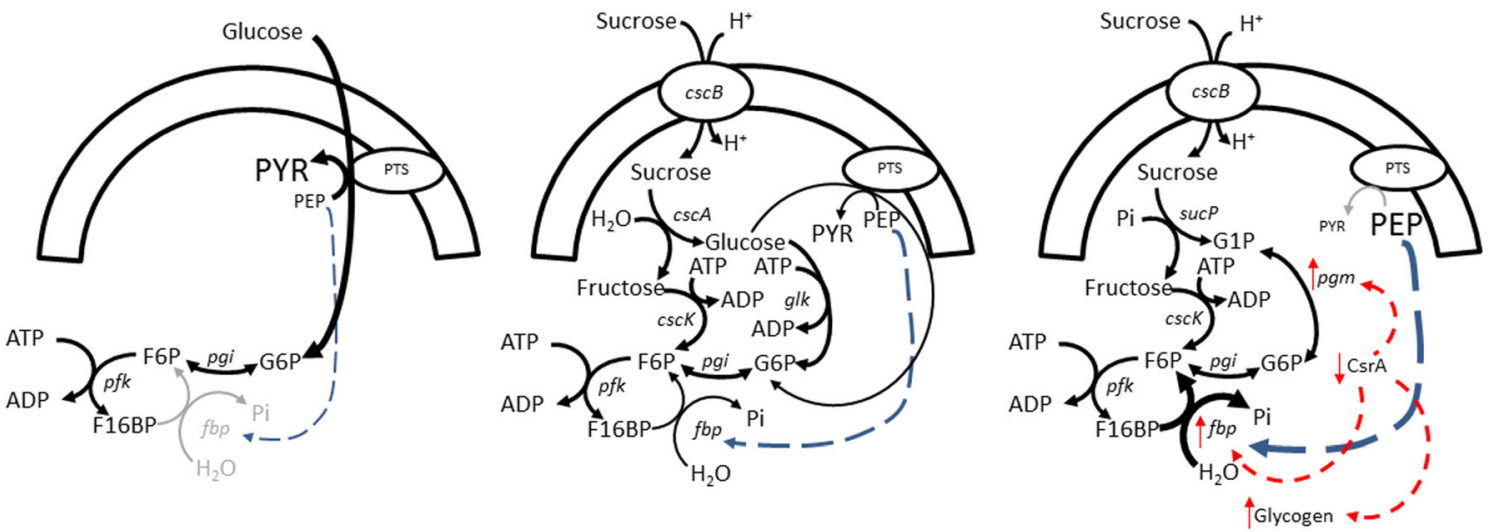

Fig. 2 Possible regulatory mechanisms leading to an increment in the flux through the futile cycle PFK/fructose-1,6-biphosphatase. The names of the intracellular enzymes and the sucrose $/ \mathrm{H}^{+}$symporter are represented with the names of their encoding genes. Left, uptake of glucose through the PTS. The concentration of PEP is small and fructose-1,6-biphosphatase is barely active. Middle, in the strain SUCHYD growing on sucrose, a fraction of the internally released glucose is phosphorylated by the PTS. However, the concentration of PEP is higher than that in cells growing on glucose and consequently the fructose-1,6-

from the oxygen-limited cultures. For comparison, the PGM activity in cells from the strain TUD69 aerobically grown on sucrose (PGM is required to survive in these conditions) was also measured. The results showed that the PGM activity was much higher in the sample taken from SUC-PHOSP compared with that from SUC-HYD and TUD69 (Table 5). This observation is consistent with the hypothesis of lower CsrA activity in the cells of SUCPHOSP, provoking higher expression of FBPase and PEP synthase, leading to higher ATP futile cycles.

Finally, the decrease in CsrA activity should also lead to a larger accumulation of glycogen. To test this second prediction, the concentrations of glycogen in SUC-HYD and SUC-PHOSP were measured. Glycogen content corresponded to $0.8 \%$ of the biomass in SUC-PHOSP and $0.1 \%$ in the case of SUC-HYD. The reported glycogen content of $E$. coli MG1655 growing on glucose in an aerobic carbon-limited chemostat at a dilution rate of $0.05 \mathrm{~h}^{-1}$ is about $0.8 \%$ (Taymaz-Nikerel et al. 2010), a value in agreement with our result for SUC-PHOSP. FBA was employed to evaluate the impact on the ATP consumption of the observed difference in glycogen content. As a result, the increase in ATP consumption to account for the biphosphatase activity is higher. Right, in the case of the strain SUCPHOSP, the activity of PTS should be absent and the concentration of PEP should be even higher leading to a stronger fructose-1,6biphosphatase activity. Still, it is likely that the activity of the CsrA protein be smaller in SUC-PHOSP leading to a higher PGM activity, a higher fructose-1,6-biphosphatase activity, and a larger accumulation of glycogen. PTS, phosphotransferase system; G6P, glucose-6-phosphate; F6P, fructose-6-phosphate; G1P, glucose-1-phosphate; F16BP, fructose-1,6biphosphate; $\mathrm{Pi}$, inorganic phosphate

observed difference in glycogen content was only 0.08 $\mathrm{mmol}_{\mathrm{ATP}} / \mathrm{g}_{\mathrm{CDW}} / \mathrm{h}$. Therefore, the difference in glycogen content does not explain the differences in biomass yields between SUC-HYD and SUC-PHOSP. Nevertheless, the higher glycogen content in SUC-PHOSP is consistent with the hypothesis of lower CsrA activity in that strain.

\section{Discussion}

There is a long background information regarding the functional characterization of the genes enabling sucrose uptake and hydrolysis from different species (Alaeddinoglu and Charles 1979; Bockmann et al. 1992; Jahreis et al. 2002; Sabri et al. 2013). Bruschi and coworkers summarized the efforts focused in the expression of such genes in E. coli strains incapable to grow on sucrose (Bruschi et al. 2012). However, to the best of our knowledge, this the first time that the energetic impact of sucrose phosphorolysis in E. coli was quantified.

Kim and co-workers reported the growth of E. coli JM-109 in M9 plates supplemented with raffinose, melibiose, or sucrose (at $40 \mathrm{~g} / \mathrm{L}$ ) after the introduction of a plasmid carrying
Table 5 Specific

phosphoglucomutase activity

\begin{tabular}{cccc}
\hline Strain & Growth conditions & Carbon source & Specific activity (U/mg soluble protein $)$ \\
\hline TUD69 & Aerobic shake flask & Sucrose & $0.074 \pm 0.012$ \\
SUC-HYD & chemostat & Sucrose & $0.013 \pm 0.002$ \\
SUC-PHOSP & chemostat & Sucrose & $9.872 \pm 0.870$ \\
\hline
\end{tabular}

$1 \mathrm{U}=\mu \mathrm{mol}_{\mathrm{NADPH}} / \mathrm{min}$ 
the putative sucP gene from Bifidobacterium longum (Kim et al. 2003). The encoded protein was semi-purified and showed remarkable similarities with other sucrose phosphorylases. Nevertheless, these authors only reported a qualitative result (growth on plates where sucrose was the sole carbon source) without reporting any growth rate. Moreover, the enzymatic assays to verify sucrose phosphorolysis were performed with synthetic analogs instead of sucrose.

In another of the few reported cases of $E$. coli growth driven by sucrose phosphorolysis, De Bruyn and co-workers replaced the chromosomal $\csc A$ gene in $E$. coli $\mathrm{W}$ by the sucP gene from Bifidobacterium adolescentis to enhance the production of UDP-glucose (derived from glucose-1-phosphate coming from sucrose phosphorolysis) (De Bruyn et al. 2015). The production of glycosylated phenolic acids derived from the UDP-glucose and externally supplied phenolic compounds indicated that sucrose phosphorolysis indeed happened.

According to Bruschi and co-workers (Bruschi et al. 2012), it is enough to express the $\csc B K A$ genes from $E$. coli $\mathrm{W}$ to observe the growth on sucrose in E. coli K-12 MG1655. Therefore, we considered not necessary to express the $\operatorname{scr} Y$ gene from the plasmid pUR400, encoding for a sucrose porin anchored in the outer membrane (Schmid et al. 1988).

Although anaerobic growth of $E$. coli $\mathrm{K}-12$ is theoretically possible without producing acetate, ethanol and lactate as long as hydrogen, acetaldehyde, and succinate are produced, the experimental results indicated that the deletions of the genes $a d h E, a d h P, l d h A$, and $m h p F$ severely limited the capacity to re-oxidize NADH, provoking a redox imbalance. Moreover, it is possible that a reduced flux from acetyl-CoA to acetate, caused by the pta deletion, affected the substrate-level production of ATP in the reaction catalyzed by the acetate kinase. Still, it is possible that the use of sucrose triggered ATPdissipation mechanisms impeding the biomass formation under anaerobic conditions.

While the redox imbalance provoked by such genetic deletions could be solved with the generation of (commercially interesting) reduced products such as ethanol, lactic acid, or polyhydroxyalkanoates, the decrease in the substrate-level production of ATP and by-product suppression is a frequent problem in anaerobic bioprocesses. Because the deletions of the $a d h E, a d h P, l d h A, p t a$, and $m h p F$ genes impeded the growth under fully anaerobic conditions, the study of the energetic impact of using sucrose was performed under continuous oxygen-limiting conditions.

The observed biomass yields were lower than expected, even considering the cost of sucrose: $\mathrm{H}^{+}$symport and the plasmid and protein burdens. Remarkably, the biomass yield of the strain SUC-PHOSP was unexpectedly smaller than that observed for the strain SUC-HYD, with a relatively larger fraction of the sucrose being fully catabolized to $\mathrm{CO}_{2}$.
Evidence was found consistent with the idea of ATP futile cycles as the reason behind the difference between the expected and the observed biomass yields. Although the FBPase activity in SUC-PHOSP was near eight times higher than that in SUC-HYD, it is known that the in vivo FBPase activity is highly regulated by allosterism (Hines et al. 2006). Therefore, with the available data, it is impossible to state that the in vivo metabolic flux through FBPase was indeed higher in SUCPHOSP. Nevertheless, we can speculate about the contributions of (i) the gene deletions, (ii) the oxygen limitation, (iii) the carbon source, (iv) the intracellular phosphate concentrations, and (v) the mechanism to break down the sucrose.

The deletion of genes encoding for enzymes involved in the by-product generation is a common practice in biotechnology (Förster and Gescher 2014; Ruiz et al. 2012). However, it has been observed a decrease in the biomass yield after the deletion of pflB (Wang et al. 2010), pflA (Matsuoka and Shimizu 2014), pta (Matsuoka and Shimizu 2014), adhE (Matsuoka and Shimizu 2014), and ldhA (Yang et al. 1999). On the other hand, De Mey and co-workers did not find differences in the maintenance ATP (around $0.088 \mathrm{~mol}$ of ATP/ C-mol biomass h) between E. coli MG1655 and its mutant lacking the genes $a c k A, p t a$, and poxB while growing anaerobically on glucose as the sole carbon source (De Mey et al. 2007).

Regarding the influence of the oxygen limitation (ii), it has been reported that the specific activities of both FBPase and PFK increase in comparison with fully aerobic conditions (Peng and Shimizu 2003). Therefore, the oxygen limitation could itself have an effect on the increase in FBPase activity. This explanation is consistent with the higher FBPase activities observed in the samples coming from the oxygen-limited cultures by comparison with the level in E. coli K-12 MG1655 growing in shaking flasks.

Regarding the carbon source employed (iii), an increased PEP to pyruvate ratio has previously been observed in E. coli MG1655 growing on sucrose (Bettenbrock et al. 2007), and it has been reported that FBPase is allosterically activated by PEP (Hines et al. 2006). Therefore, the growth on sucrose could trigger an increment in the concentration of PEP leading to an increment in the FBPase activity. Because the expression of FBPase is itself affected by the oxygen limitation, the combination of the use of sucrose with oxygen limitation could result in even larger FBPase activities compared with the levels registered growing aerobically on glucose or acetate, explaining the lower biomass yields observed in both SUCHYD and SUC-PHOSP by comparison with the expected values.

None of the previous discussed factors, however, explains the unexpected lower biomass yield observed in SUC-PHOSP by comparison with SUC-HYD. One of the factors that could be indeed different between these strains, leading to different network operations, is the intracellular free phosphate concentrations (iv). Phosphate is a substrate for the reaction catalyzed 
by the SucP and, at the same time, it is one of the products of the reaction catalyzed by the FBPase. Thus, it is possible that in those cells with higher FBPase activity, the released phosphate had a positive impact on the rate of sucrose phosphorolysis, probably easing a metabolic bottleneck. Another source of phosphate could be the flow through the methylglyoxal pathway. In this pathway, one dihydroxyacetone-phosphate is converted into pyruvate and inorganic phosphate is realized, while no ATP is produced. In that case, although more phosphate is released, the ATP yield is affected. Moreover, methylglyoxal toxicity could have additional negative effects.

Another process affected by the cytoplasmic phosphate concentration is the relative use of water or phosphate as nucleophile in the sucrose breakdown catalyzed by the SucP. However, if water fully replaced phosphate during the sucrose breakdown in SUC-PHOSP, we should have seen a biomass yield similar to SUC-HYD, but it was not the case. Still, it is possible that different intracellular concentrations of free phosphate in these two strains provoked different $\mathrm{H}^{+}$:ATP stoichiometries in the proton pumps (D'Alessandro et al. 2008). Nevertheless, given the multiple binding interactions between phosphate ions and intracellular molecules, the accurate measuring of cytoplasmic free phosphate concentrations is extremely challenging and requires significant additional effort beyond the scope of this research.

Next to the potential differences in the intracellular free phosphate concentrations, the differences between the mechanisms to break down the sucrose in SUC-HYD and SUC-PHOSP (v) could result in different metabolomics profile. Because PTS has the capacity to phosphorylate both extracellular and intracellular glucose (Postma et al. 1993), the PTS activity was probably higher in SUC-HYD than in SUC-PHOSP, leading to lower concentration of PEP in SUC-HYD and, consequently, a lower FBPase activity by comparison with SUC-PHOSP (Fig. 2). A report showing that deletion of the ptsI-ptsH-crr genes in E. coli increased the concentration of PEP (Flores and Xiao 1996) is consistent with this explanation.

It was finally found evidence pointing to different levels of CsrA activities in SUC-HYD and SUC-PHOSP. Although a decrease in CsrA activity could improve PGM activity, making easier the assimilation of carbon in SUC-PHOSP, it could also increase the activities of futile ATP cycle-related enzymes such as FBPase and PEP synthase.

Overall, our results confirm that the implementation of sucrose consumption and reduction of by-product in E. coli K12 is technically easy. However, they also showed an unexpected increase in ATP expenses under such conditions, being larger in the strain expressing a sucrose phosphorylase. The found evidence points to the operation of an ATP futile cycle due to the simultaneous operation of the phosphofructokinase(s) and the FBPase. One theoretically possible way to eliminate this futile cycle is the deletion of the genes $p f k A$ and $p f k B$, enforcing the glycolytic flow through reactions belonging to the Entner-Doudoroff pathway or the pentosephosphate pathway (Hollinshead et al. 2016). However, this approach should reduce the ATP yield. In that case, the only way to increase the ATP yield is with the further implementation of some heterologous glycolytic pathway, such as the bifid shunt. On the other hand, the deletion of the genes $f b p$ and $g l p X$, encoding for fructose-1,6-biphosphatases, seems to be an easier solution but the resulting organisms will be unable to survive in gluconeogenic conditions. Therefore, the best way to suppress this potential ATP futile cycle will depend on the specific application.

Anyways, it is extremely complicated to eliminate all the possibilities for futile cycles and probably is not recommendable either. Next to the case discussed above, futile cycles can be established by the simultaneous activity of phosphoenolpyruvate synthase (EC 2.7.9.2) and pyruvate kinase (EC 2.7.1.40) (Patnaik et al. 1992) or phosphoenolpyruvate carboxykinase (EC 4.1.1.49) and phosphoenolpyruvate carboxylase (EC 4.1.1.31) (Chao and Liao 1994) among others. ATP dissipation seems to be present in many organisms, contributing to the adjustment between the rates of catabolic supply and anabolic demand (Russell 2007) and could be also important as a defense mechanism against oxidative stress (Adolfsen and Brynildsen 2015).

With the obtained data, it is not possible to distinguish the individual contributions of the by-product suppression, the oxygen limitation, and the use of sucrose to the observed increment in the ATP expense. Therefore, further studies focused in the observation of the individual effects of these factors are required.

It is surprising that, despite the frequent observation of decrease in the biomass yields after the deletions of genes involved in by-product generation, the mechanism(s) explaining the fall in the biomass yields are seldom discussed. Wang and co-workers proposed that ATP dissipation was probably a response to a relative excess of ATP respect to the amino acids and other building blocks in the mutant lacking $p f l B$ (Wang et al. 2010). It is possible that those gene deletions provoked metabolic bottlenecks, triggering the expression of genes involved in futile cycles. As a matter of fact, in the cases of the deletion of pflA, pta, and $a d h E$, increments in the concentration of several central pathway metabolites were observed, including fructose-1,6-bisphosphate (Matsuoka and Shimizu 2014), which is widely acknowledged as a signaling metabolite (Kochanowski et al. 2013).

Regarding the potential effect of oxygen limitation in the observed decrease of the ATP yields, it seems interesting to study the impact on the ATP yields of the introduction of production pathways capable to re-oxidize the NADH under anaerobic conditions. One possibility could be the introduction of a NADHconsuming polyhydroxybutyrate pathway (de Las Heras et al. 2016; Ling et al. 2018).

Finally, the metabolic impact of the use of sucrose in E. coli MG1655 derivatives requires attention. While studying the sucrose metabolism of an $E$. coli strain naturally capable to grow on sucrose, Jahreis and co-workers found evidence indicating an 
evolutionary recent acquisition of the genes enabling the use of this disaccharide (Jahreis et al. 2002). The catabolism of sucrose using a hydrolase or a phosphorylase implies intracellular concentrations of fructose and glucose (or glucose-1-phosphate) larger than the expected for the catabolism of glucose. It is possible that an increment in the concentration of sugar phosphates induces the (energetically less efficient) methylglyoxal pathway. To avoid this situation, an increment in the phosphoglucomutase activity could accelerate the funneling of carbon into the Embden-Meyerhof pathway, preventing the accumulation of sugar phosphates. Thus, further studies focused on the changes in the metabolome when the carbon source shifts from glucose to sucrose should help us to understand the mechanisms behind the unexpected decrease in biomass yield after the implementation of sucrose consumption in E. coli K-12.

Authors' contributions K.O. and S.A.W. conceived the research. K.O. wrote the manuscript, including comments and critics from other authors. A.F. contributed important ideas in the experimental design, performed most of the experiments, and critically reviewed the manuscript. K.O. and M.I. Velasco performed some experiments and critically reviewed the manuscript. M.C.M.v.L. contributed to the general design of the research and reviewed critically the manuscript.

Funding information This work was supported by the joint research program NWO-FAPESP from The Netherlands Organization for Scientific Research (NWO) and São Paulo Research Foundation (FAPESP) with grant number BRAZIL.2013.018.

The contributions of Karel Olavarria and M.C.M. van Loosdrecht were supported by SIAM Gravitation Grant (024.002.002) of the Netherlands Ministry of Education, Culture and Science (OCW) and NWO.

\section{Compliance with ethical standards}

Competing interests The authors declare that they have no competing interests.

Ethics approval and consent to participate The research was performed according with the ethical and biosafety standards approved in our institution. The authors gave their consent to participate in the research and the publication of the results.

Open Access This article is distributed under the terms of the Creative Commons Attribution 4.0 International License (http:// creativecommons.org/licenses/by/4.0/), which permits unrestricted use, distribution, and reproduction in any medium, provided you give appropriate credit to the original author(s) and the source, provide a link to the Creative Commons license, and indicate if changes were made.

\section{References}

Adolfsen KJ, Brynildsen MP (2015) Futile cycling increases sensitivity toward oxidative stress in Escherichia coli. Metab Eng 29:26-35. https://doi.org/10.1016/j.ymben.2015.02.006

Alaeddinoglu NG, Charles HP (1979) Transfer of a gene for sucrose utilization into Escherichia coli $\mathrm{K} 12$, and consequent failure of expression of genes for D-serine utilization. J Gen Microbiol 110(1):47-59. https://doi.org/10.1099/00221287-110-1-47

Axe DD, Bailey JE (1995) Transport of lactate and acetate through the energized cytoplasmic membrane of Escherichia coli. Biotechnol Bioeng 47(1):8-19. https://doi.org/10.1002/bit.260470103

Bettenbrock K, Sauter T, Jahreis K, Kremling A, Lengeler JW, Gilles ED (2007) Correlation between growth rates, EIIACrr phosphorylation, and intracellular cyclic AMP levels in Escherichia coli K-12. J Bacteriol 189(19):6891-6900. https://doi.org/10.1128/jb.00819-07

Bockmann J, Heuel H, Lengeler JW (1992) Characterization of a chromosomally encoded, non-PTS metabolic pathway for sucrose utilization in Escherichia coli EC3132. Mol Gen Genet 235(1):22-32

Bruschi M, Boyes SJ, Sugiarto H, Nielsen LK, Vickers CE (2012) A transferable sucrose utilization approach for non-sucrose-utilizing Escherichia coli strains. Biotechnol Adv 30(5):1001-1010. https:// doi.org/10.1016/j.biotechadv.2011.08.019

Chao YP, Liao JC (1994) Metabolic responses to substrate futile cycling in Escherichia coli. J Biol Chem 269(7):5122-5126

Cueto-Rojas HF, van Maris AJA, Wahl SA, Heijnen JJ (2015) Thermodynamics-based design of microbial cell factories for anaerobic product formation. Trends Biotechnol 33(9):534-546. https:// doi.org/10.1016/j.tibtech.2015.06.010

D'Alessandro M, Turina P, Melandri BA (2008) Intrinsic uncoupling in the ATP synthase of Escherichia coli. Biochim Biophys Acta 1777(12):1518-1527. https://doi.org/10.1016/j.bbabio.2008.09.011

Datsenko KA, Wanner BL (2000) One-step inactivation of chromosomal genes in Escherichia coli K-12 using PCR products. Proc Natl Acad Sci U S A 97(12):6640-6645. https://doi.org/10.1073/pnas. 120163297

De Bruyn F, De Paepe B, Maertens J, Beauprez J, De Cocker P, Mincke S, Stevens C, De Mey M (2015) Development of an in vivo glucosylation platform by coupling production to growth: production of phenolic glucosides by a glycosyltransferase of Vitis vinifera. Biotechnol Bioeng 112(8):1594-1603. https://doi.org/10.1002/bit. 25570

de Kok S, Yilmaz D, Suir E, Pronk JT, Daran JM, van Maris AJ (2011) Increasing free-energy (ATP) conservation in maltose-grown Saccharomyces cerevisiae by expression of a heterologous maltose phosphorylase. Metab Eng 13(5):518-526. https://doi.org/10.1016/ j.ymben.2011.06.001

de Las Heras AM, Portugal-Nunes DJ, Rizza N, Sandstrom AG, GorwaGrauslund MF (2016) Anaerobic poly-3-D-hydroxybutyrate production from xylose in recombinant Saccharomyces cerevisiae using a NADH-dependent acetoacetyl-CoA reductase. Microb Cell Fact 15(1):197. https://doi.org/10.1186/s12934-016-0598-0

De Mey M, Lequeux GJ, Beauprez JJ, Maertens J, Van Horen E, Soetaert WK, Vanrolleghem PA, Vandamme EJ (2007) Comparison of different strategies to reduce acetate formation in Escherichia coli. Biotechnol Prog 23(5):1053-1063. https://doi.org/10.1021/ bp070170g

Dikicioglu D, Kirdar B, Oliver SG (2015) Biomass composition: the "elephant in the room" of metabolic modelling. Metabolomics 11(6):1690-1701. https://doi.org/10.1007/s11306-015-0819-2

Flores N, Xiao J (1996) Berry a, Bolivar F, Valle F (1996) Pathway engineering for the production of aromatic compounds in Escherichia coli. Nat Biotechnol 14:620-623. https://doi.org/10. 1038/nbt0596-620

Förster AH, Gescher J (2014) Metabolic engineering of Escherichia coli for production of mixed-acid fermentation end products. Front Bioeng Biotechnol 2:16-16. https://doi.org/10.3389/fbioe.2014. 00016

Gonzalez JE, Long CP, Antoniewicz MR (2017) Comprehensive analysis of glucose and xylose metabolism in Escherichia coli under aerobic and anaerobic conditions by (13)C metabolic flux analysis. Metab Eng 39:9-18. https://doi.org/10.1016/j.ymben.2016.11.003 
Harris RM, Webb DC, Howitt SM, Cox GB (2001) Characterization of PitA and PitB from Escherichia coli. J Bacteriol 183(17):50085014. https://doi.org/10.1128/JB.183.17.5008-5014.2001

Hines JK, Fromm HJ, Honzatko RB (2006) Novel allosteric activation site in Escherichia coli fructose-1,6- bisphosphatase. J Biol Chem. 281(27):18386-18393. https://doi.org/10.1074/jbc.M602553200

Hoefel T, Faust G, Reinecke L, Rudinger N, Weuster-Botz D (2012) Comparative reaction engineering studies for succinic acid production from sucrose by metabolically engineered Escherichia coli in fed-batch-operated stirred tank bioreactors. Biotechnol J 7(10): 1277-1287. https://doi.org/10.1002/biot.201200046

Hollinshead WD, Rodriguez S, Martin HG, Wang G, Baidoo EE, Sale KL, Keasling JD, Mukhopadhyay A, Tang YJ (2016) Examining Escherichia coli glycolytic pathways, catabolite repression, and metabolite channeling using $\Delta p f k$ mutants. Biotechnol Biofuels 9:212. https://doi.org/10.1186/s13068-016-0630-y

Jahreis K, Bentler L, Bockmann J, Hans S, Meyer A, Siepelmeyer J, Lengeler JW (2002) Adaptation of sucrose metabolism in the Escherichia coli wild-type strain EC3132. J Bacteriol 184(19): 5307-5316

Jantama K, Zhang X, Moore JC, Shanmugam KT, Svoronos SA, Ingram LO (2008) Eliminating side products and increasing succinate yields in engineered strains of Escherichia coli C. Biotechnol Bioeng 101(5):881-893. https://doi.org/10.1002/bit.22005

Jian J, Zhang SQ, Shi ZY, Wang W, Chen GQ, Wu Q (2010) Production of polyhydroxyalkanoates by Escherichia coli mutants with defected mixed acid fermentation pathways. Appl Microbiol Biotechnol 87(6):2247-2256. https://doi.org/10.1007/s00253-0102706-0

Kim MJ, Kwon T, Lee HJ, Kim KH, Chung DK, Ji GE, Byeon ES, Lee $\mathrm{JH}$ (2003) Cloning and expression of sucrose phosphorylase gene from Bifidobacterium longum in E. coli and characterization of the recombinant enzyme. Biotechnol Lett 25(15):1211-1217. https:// doi.org/10.1023/A:1025035320983

Kochanowski K, Volkmer B, Gerosa L, van Rijsewijk B, Schmidt A, Heinemann M (2013) Functioning of a metabolic flux sensor in Escherichia coli. Proc Natl Acad Sci U S A 110(3):1130-1135. https://doi.org/10.1073/pnas.1202582110

Lamarche MG, Wanner BL, Crépin S, Harel J (2008) The phosphate regulon and bacterial virulence: a regulatory network connecting phosphate homeostasis and pathogenesis. FEMS Microbiol Rev 32(3):461-473. https://doi.org/10.1111/j.1574-6976.2008.00101.x

Ling C, Qiao GQ, Shuai BW, Olavarria K, Yin J, Xiang RJ, Song KN, Shen YH, Guo Y, Chen GQ (2018) Engineering NADH/NAD(+) ratio in Halomonas bluephagenesis for enhanced production of polyhydroxyalkanoates (PHA). Metab Eng 49:275-286. https:// doi.org/10.1016/j.ymben.2018.09.007

Marques WL, Raghavendran V, Stambuk BU, Gombert AK (2016) Sucrose and Saccharomyces cerevisiae: a relationship most sweet. FEMS Yeast Res 16(1):fov107. https://doi.org/10.1093/femsyr/ fov107

Marques WL, Mans R, Henderson RK, Marella ER, Horst JT, Hulster E, Poolman B, Daran JM, Pronk JT, Gombert AK, van Maris AJA (2018) Combined engineering of disaccharide transport and phosphorolysis for enhanced ATP yield from sucrose fermentation in Saccharomyces cerevisiae. Metab Eng 45:121-133. https://doi.org/ 10.1016/j.ymben.2017.11.012

Matsuoka Y, Shimizu K (2014) Metabolic flux analysis for Escherichia coli by flux balance analysis. Methods Mol Biol 1191:237-260. https://doi.org/10.1007/978-1-4939-1170-7_15

Mukherjee K, Narindoshvili T, Raushel FM (2018) Discovery of a kojibiose phosphorylase in Escherichia coli K-12. Biochemistry 57(19):2857-2867. https://doi.org/10.1021/acs.biochem.8b00392

Patnaik R, Roof WD, Young RF, Liao JC (1992) Stimulation of glucose catabolism in Escherichia coli by a potential futile cycle. J Bacteriol 174(23):7527-7532
Peng L, Shimizu K (2003) Global metabolic regulation analysis for Escherichia coli K12 based on protein expression by 2 dimensional electrophoresis and enzyme activity measurement. Appl Microbiol Biotechnol 61(2):163-178. https://doi.org/10. 1007/s00253-002-1202-6

Porro D, Branduardi P, Sauer M, Mattanovich D (2014) Old obstacles and new horizons for microbial chemical production. Curr Opin Biotechnol 30:101-106. https://doi.org/10.1016/j.copbio.2014.06. 009

Postma PW, Lengeler JW, Jacobson GR (1993) Phosphoenolpyruvate: carbohydrate phosphotransferase systems of bacteria. Microbiol Rev 57(3):543-594

Roe AJ, O'Byrne C, McLaggan D, Booth IR (2002) Inhibition of Escherichia coli growth by acetic acid: a problem with methionine biosynthesis and homocysteine toxicity. Microbiology 148(Pt 7): 2215-2222. https://doi.org/10.1099/00221287-148-7-2215

Ruiz JA, de Almeida A, Godoy MS, Mezzina MP, Bidart GN, Méndez BS, Pettinari MJ, Nikel PI (2012) Escherichia coli redox mutants as microbial cell factories for the synthesis of reduced biochemicals. Comput Struct Biotechnol J 3(4):e201210019. https://doi.org/10. 5936/csbj.201210019

Russell JB (2007) The energy spilling reactions of bacteria and other organisms. J Mol Microbiol Biotechnol 13(1-3):1-11. https://doi. org/10.1159/000103591

Sabnis NA, Yang H, Romeo T (1995) Pleiotropic regulation of central carbohydrate-metabolism in Escherichia coli via the gene Csra. J Biol Chem. 270(49):29096-29104. https://doi.org/10.1074/jbc.270. 49.29096

Sabri S, Nielsen LK, Vickers CE (2013) Molecular control of sucrose utilization in Escherichia coli W, an efficient sucrose-utilizing strain. Appl Environ Microbiol 79(2):478-487. https://doi.org/10.1128/ aem.02544-12

Sahin-Toth M, Lengyel Z, Tsunekawa H (1999) Cloning, sequencing, and expression of $\csc A$ invertase from Escherichia coli B-62. Can J Microbiol 45(5):418-422

Schellenberger J, Que R, Fleming RMT, Thiele I, Orth JD, Feist AM, Zielinski DC, Bordbar A, Lewis NE, Rahmanian S, Kang J, Hyduke DR, Palsson B (2011) Quantitative prediction of cellular metabolism with constraint-based models: the COBRAToolbox v2.0. Nat Protoc 6:1290-1307

Schmid K, Ebner R, Altenbuchner J, Schmitt R, Lengeler JW (1988) Plasmid-mediated sucrose metabolism in Escherichia coli K12: mapping of the $s c r$ genes of pUR400. Mol Microbiol 2(1):1-8

Schuetz R, Kuepfer L, Sauer U (2007) Systematic evaluation of objective functions for predicting intracellular fluxes in Escherichia coli. Mol Syst Biol 3:119. https://doi.org/10.1038/msb4100162

Soini J, Ukkonen K, Neubauer P (2008) High cell density media for Escherichia coli are generally designed for aerobic cultivations consequences for large-scale bioprocesses and shake flask cultures. Microb Cell Fact 7:26. https://doi.org/10.1186/1475-2859-7-26

Taymaz-Nikerel H, Borujeni AE, Verheijen PJ, Heijnen JJ, van Gulik WM (2010) Genome-derived minimal metabolic models for Escherichia coli MG1655 with estimated in vivo respiratory ATP stoichiometry. Biotechnol Bioeng 107(2):369-381. https://doi.org/ $10.1002 /$ bit.22802

Tseng H-C, Martin CH, Nielsen DR, Jones Prather KL (2009) Metabolic engineering of Escherichia coli for enhanced production of (R)-and (S)-3-hydroxybutyrate. Appl Environ Microbiol 75(10):3137-3145. https://doi.org/10.1128/aem.02667-08

Tsunekawa H, Azuma S, Okabe M, Okamoto R, Aiba S (1992) Acquisition of a sucrose utilization system in Escherichia coli K12 derivatives and its application to industry. Appl Environ Microbiol 58(6):2081-2088

van Gulik WM, Heijnen JJ (1995) A metabolic network stoichiometry analysis of microbial growth and product formation. Biotechnol Bioeng 48(6):681-698. https://doi.org/10.1002/bit.260480617 
Verduyn C, Postma E, Scheffers WA, van Dijken JP (1992) Effect of benzoic acid on metabolic fluxes in yeasts: a continuous-culture study on the regulation of respiration and alcoholic fermentation. Yeast 8(1992):501-517. https://doi.org/10.1007/bf00270792

Wang Q, Ou MS, Kim Y, Ingram LO, Shanmugam KT (2010) Metabolic flux control at the pyruvate node in an anaerobic Escherichia coli strain with an active pyruvate dehydrogenase. Appl Environ Microbiol 76(7):2107-2114. https://doi.org/10.1128/AEM.02545-09

Wang Y, Tian T, Zhao J, Wang J, Yan T, Xu L, Liu Z, Garza E, Iverson A, Manow R, Finan C, Zhou S (2012) Homofermentative production of D-lactic acid from sucrose by a metabolically engineered Escherichia coli. Biotechnol Lett 34(11):2069-2075. https://doi. org/10.1007/s10529-012-1003-7

Willsky GR, Malamy MH (1980) Characterization of two genetically separable inorganic phosphate transport systems in Escherichia coli. J Bacteriol 144(1):356-365
Yang Y-T, San K-Y, Bennett GN (1999) Redistribution of metabolic fluxes in Escherichia coli with fermentative lactate dehydrogenase overexpression and deletion. Metab Eng 1(2):141-152. https://doi. org/10.1006/mben.1998.0111

Zhang YH, Lynd LR (2005) Cellulose utilization by Clostridium thermocellum: bioenergetics and hydrolysis product assimilation. Proc Natl Acad Sci U S A 102(20):7321-7325. https://doi.org/10. 1073/pnas.0408734102

Publisher's note Springer Nature remains neutral with regard to jurisdictional claims in published maps and institutional affiliations. 